PROCEEDINGS OF THE

AMERICAN MATHEMATICAL SOCIETY

Volume 135, Number 6, June 2007, Pages 1651-1657

S 0002-9939(07)08707-2

Article electronically published on January 9, 2007

\title{
ON CURTIS' THEOREM ABOUT FINITE OCTONIONIC LOOPS
}

\author{
PAUL BODDINGTON AND DMITRIY RUMYNIN
}

(Communicated by Jonathan I. Hall)

\begin{abstract}
We prove a stronger version of Curtis' classification theorem of finite subloops of the Cayley octonions $\mathbb{O}$.
\end{abstract}

It is well known that all finite subgroups of $\mathbb{C}^{*}$ are cyclic. Moreover, for each $n$ there exists a unique cyclic subgroup of $\mathbb{C}^{*}$ of order $n$.

Finite subgroups of $\mathbb{H}^{*}$ can be classified up to the action of automorphism group $S_{3}(\mathbb{R})$ of $\mathbb{H}$. The problem is equivalent to the classification of finite subgroups of $S L_{2}(\mathbb{C})$. The solution of this problem is usually attributed to Jordan [1, although we have not managed to acquire the cited paper. A more elegant answer is given by McKay 22. He associates to each finite subgroup its McKay label, a simply-laced Dynkin diagram $A_{n}, D_{n}, E_{6}, E_{7}, E_{8}$.

In this note we prove the following classification theorem. Let $G=\operatorname{Aut}(\mathbb{O})$ be the compact group of type $G_{2}$.

Theorem 1. Let $\Gamma$ be a finite subloop of the multiplicative loop $\mathbb{O}^{*}=\mathbb{O} \backslash\{0\}$ of the Cayley algebra $\mathbb{O}$. Then it is either associative or the double of a non-commutative associative subloop or the group of units in the octavian integers. Two finite subloops $\Gamma$ and $\Delta$ are conjugate by the action of $G$ if and only if one the following three statements hold:

1) both $\Gamma$ and $\Delta$ are associative and have the same McKay label;

2) both $\Gamma$ and $\Delta$ are doubles of non-commutative associative loops with the same McKay label;

3) both $\Gamma$ and $\Delta$ are unit loops of octavian integers.

A similar theorem was proved by R. T. Curtis 3 but the manuscript has been unpublished for 35 years. We have come by the result independently, and our proof is different. Our theorem is stronger since our classification is up to $G$-action, while Curtis' classification is up to an isomorphism. On the other hand, Curtis gives some additional information such as generators and relations for all finite loops [4]. We would like to thank Robert T. Curtis for providing a copy of his manuscript and Jeremy Gray for valuable discussions.

Received by the editors July 19, 2005 and, in revised form, February 24, 2006.

2000 Mathematics Subject Classification. Primary 17D05; Secondary 17B20.

Key words and phrases. Octonions, quaternions, loop, root system.

(C)2007 American Mathematical Society Reverts to public domain 28 years from publication 


\section{Subloops as RoOt Systems}

Dynkin labels $A-D-E$ are attached to finite subgroups of $\mathbb{H}$ using McKay correspondence [2, 5]. However, there is a second connection between finite subgroups and root systems [6, Lemma 2.13]. Any finite subgroup of $\mathbb{H}$ containing -1 is a root system with all roots of unit length as summarized in the following table.

\begin{tabular}{|c||c|c|c|c|c|}
\hline McKay label & $A_{2 n-1}$ & $D_{n}$ & $E_{6}$ & $E_{7}$ & $E_{8}$ \\
\hline Root System & $I_{2}(n)$ & $I_{2}(n-2)^{2}$ & $D_{4}$ & $F_{4}$ & $H_{4}$ \\
\hline Order & $2 n$ & $4(n-2)$ & 24 & 48 & 120 \\
\hline
\end{tabular}

We establish a similar result for finite subloops of $\mathbb{O}$ in this section. We start by reminding the reader of a construction of octonions.

Let $I, J$ and $K$ be the standard quaternions of $\mathbb{H}$ with $I J=K$. Then

$$
\mathbb{O}=\mathbb{H} \oplus \omega \mathbb{H}, \quad(a+\omega b)(c+\omega d)=\left(a c-d b^{*}\right)+\omega\left(a^{*} d+c b\right) .
$$

Let us introduce unit octonions $e_{i}$ by $e_{0}=1, e_{1}=I, e_{2}=J, e_{3}=\omega, e_{4}=K, e_{5}=$ $-\omega J, e_{6}=\omega K, e_{7}=\omega I$ following the standard convention $e_{i} e_{i+1}=e_{i+3}, i>0$. We write $\mathbb{O}=\mathbb{R} \oplus \mathbb{L}$, where $\mathbb{L}$ is the seven-dimensional space of imaginary octonions. Projections to $\mathbb{R}$ and $\mathbb{L}$ are denoted $\operatorname{Re}$ and $\operatorname{Im}$. Then $x^{*}=\operatorname{Re}(x)-\operatorname{Im}(x), x \in \mathbb{O}$, is the conjugation; $n(x)=x x^{*}$ is the quadratic form with the Euclidean inner product $\langle x, y\rangle=\left(x y^{*}+y x^{*}\right) / 2=\operatorname{Re}\left(x y^{*}\right)$. For each $x \in \mathbb{O}^{*}=\mathbb{O} \backslash\{0\}$ we have a reflection

$$
S_{x}: \mathbb{O} \rightarrow \mathbb{O}, \quad S_{x}(y)=y-2 \frac{\langle x, y\rangle}{\langle x, x\rangle} x .
$$

We are going to skip unnecessary parentheses, such as $x y^{*} x=\left(x y^{*}\right) x=x\left(y^{*} x\right)$ in the next lemma. It holds since any 2-generated subloop of $\Gamma$ is associative, which follows from Artin's theorem that any 2-generated subalgebra of $\mathbb{O}$ is associative [7. 6.8].

Lemma 1. If $x \in \mathbb{O}$ such that $n(x)=1$, then $S_{x}(y)=-x y^{*} x$ for each $y \in \mathbb{O}$.

Proof. We observe that

$S_{x}(y)=\left(S_{x}(y) x^{*}\right) x=\left(y x^{*}-2\langle x, y\rangle x x^{*}\right) x=\left(y x^{*}+x y^{*}-x y^{*}-2\langle x, y\rangle\right) x=-x y^{*} x$.

Recall that a root system is a finite subset $\Phi$ of a euclidean space such that $S_{x}(\Phi)=\Phi$ and $\Phi \cap \mathbb{R} x=\{x,-x\}$ for all $x \in \Phi[6$, 1.2]. Since we do not set any requirement on the values of Cartan numbers $2\langle x, y\rangle /\langle x, x\rangle$, root lengths enjoy greater freedom, for instance, all roots of system $F_{4}$ can be of unit length in the next lemma. If $\Phi=\Phi_{1} \cup \Phi_{2}$ are roots systems and $\Phi_{1} \perp \Phi_{2}$, then we say $\Phi$ is a direct sum of $\Phi_{1}$ and $\Phi_{2}$. Irreducible systems are those without non-trivial direct sum decompositions; their classification follows the classification of finite Coxeter groups [6].

Lemma 2. If $\Gamma$ is finite subloop of $\mathbb{O}$ and $-1 \in \Gamma$, then $\Gamma$ is a root system with all roots of unit length. If $\Gamma=\Gamma_{1} \oplus \Gamma_{2} \oplus \ldots \oplus \Gamma_{k}$ is a decomposition into irreducible root systems with $1 \in \Gamma_{1}$, then $\Gamma_{1} \cong \Gamma_{2} \cong \ldots \cong \Gamma_{k}$ as root systems. Moreover, $\Gamma_{1}$ is a subloop, and any subloop of $\Gamma$ containing $\Gamma_{1}$ is of the form $\bigoplus \Gamma_{i}$. 
Proof. Each element $g \in \Gamma$ has a finite order. In particular, as soon as $g^{k}=g^{m}$, $k>m$, it follows that $g^{k-m}=1$. This implies that $n(g)=1$, and the first statement follows from the previous lemma.

For each $g \in \Gamma_{i}$ we have that $\Gamma=g \Gamma_{1} \oplus g \Gamma_{2} \oplus \ldots \oplus g \Gamma_{k}$. Thus, $g \Gamma_{1}=\Gamma_{i}$ and all irreducible systems have the same type. Finally, if $\Delta \supseteq \Gamma_{1}$ is a subloop of $\Gamma$, then $\Delta=\bigcup_{g \in \Delta} g \Gamma_{1}$, and each $g \Gamma_{1}$ must be one of irreducible subsystems $\Gamma_{i}$.

We will need the following standard fact.

Lemma 3. $\mathbb{R}, \mathbb{C}, \mathbb{H}$ and $\mathbb{O}$ are the only subalgebras of $\mathbb{O}$ (over the field $\mathbb{R}$ ) up to an algebra automorphism.

Proof. By Hurwitz's theorem [7, 6.4], any subalgebra is isomorphic to one of $\mathbb{R}$, $\mathbb{C}, \mathbb{H}$ or $\mathbb{O}$. It remains to show that any two isomorphic subalgebras are conjugate under algebra automorphism of $\mathbb{O}$. It is trivial for $\mathbb{R}$ and $\mathbb{O}$.

Let $\mathbb{H}$ and $\mathbb{H}^{\prime}$ be two subalgebras of quaternions of $\mathbb{O}$. Choosing imaginary units $\omega$ and $\omega^{\prime}$ gives the decomposition

$$
\mathbb{O}=\mathbb{H} \oplus \omega \mathbb{H}=\mathbb{H}^{\prime} \oplus \omega^{\prime} \mathbb{H}^{\prime},
$$

with the same multiplication formula (11) [7, 6.3]. Choosing an isomorphism $\psi$ : $\mathbb{H} \rightarrow \mathbb{H}^{\prime}$ gives the required automorphism $a+\omega b \mapsto \psi(a)+\omega^{\prime} \psi(b)$.

The proof for $\mathbb{C}$ is similar. First, we embed $\mathbb{C} \subseteq \mathbb{H}$ and $\mathbb{C}^{\prime} \subseteq \mathbb{H}^{\prime}$. Then, we find an automorphism $\psi$ sending $\mathbb{H}$ to $\mathbb{H}^{\prime}$. Finally, we derive an automorphism of $\mathbb{H}^{\prime}$ that sends $\psi(\mathbb{C})$ to $\mathbb{C}^{\prime}$.

Let us clarify the minus identity assumption.

Lemma 4. If $\Gamma$ is a finite subloop of $\mathbb{O}$ without -1 , then $\Gamma$ is a cyclic group of odd order.

Proof. If $-1 \notin \Gamma$, then the order of each $g \in \Gamma$ is odd. Let $\Delta$ be a 2-generated subloop of $\Gamma$. It is associative by Artin's theorem [7, 6.8]. Thus, $\Delta$ spans a subalgebra contained in $\mathbb{H}$ by Lemma 3 , Furthermore, the order of $\Delta$ is odd. It follows from the classification of finite subgroups of $\mathbb{H}$ that $\Delta$ is a cyclic group. Thus, $\Gamma$ is commutative. By Lemma 3 , the span of $\Gamma$ lies in $\mathbb{C}$, and the lemma follows.

Let us summarize the discussion in this section.

Lemma 5. Let $\Gamma$ be a finite subloop of $\mathbb{O}$. If $\Gamma$ is commutative, then $\Gamma$ is a cyclic subgroup of a copy of $\mathbb{C}$ in $\mathbb{O}$. If $\Gamma$ is noncommutative and associative, then $\Gamma$ spans a copy of $\mathbb{H}$ in $\mathbb{O}$ and forms a root system of rank 4 . If $\Gamma$ is nonassociative, then $\Gamma$ spans $\mathbb{O}$ and forms a root system of rank 8.

Proof. It immediately follows from previous lemmas.

Lemma 5 tells us that the classification of associative subloops of $\mathbb{O}$ is equivalent to the classification of subgroups of $\mathbb{H}$. This is already known. The classification of non-associative subloops will be finished by Lemma 8 stating that a non-associative subloop is uniquely determined (up to an action of $G$ ) by the type of its root system. Now we know that such a subloop is a root system of rank 8 with irreducible components of the same type. Thus, it has to be on the following list: $A_{1}^{8}, I_{2}(n)^{4}(n>2)$, $A_{4}^{2}, B_{4}^{2}, D_{4}^{2}, F_{4}^{2}, H_{4}^{2}, A_{8}, B_{8}, D_{8}, E_{8}$. 
Before proceeding further, let us make a technical observation about $\Gamma^{(n)}$, the set of elements of order $n$ in $\Gamma$. We note that the sets $\Gamma^{(n)}$ can be characterised in terms of the root system

$$
\begin{gathered}
\Gamma^{(4)}=\left\{x \in \Gamma \mid x^{2}=-1\right\}=\{x \in \Gamma \mid \operatorname{Re}(x)=0\}=\left\{x \in \Gamma \mid x \perp 1_{\mathbb{O}}\right\}, \\
\Gamma^{(n)}=\left\{x \in \Gamma \mid\left\langle x, 1_{\mathbb{O}}\right\rangle=\operatorname{Re}(x)=\cos k \frac{2 \pi}{n}, k \text { and } n \text { are relatively prime }\right\} .
\end{gathered}
$$

\section{EXISTENCE}

In this section we establish the existence of loops that form certain root systems.

2.1. Subloops of type $I_{2}(n)^{4}, D_{4}^{2}, F_{4}^{2}, H_{4}^{2}$. If $\Delta$ is finite subgroup of $\mathbb{H}^{*}$, then $\Gamma=\Delta \cup \omega \Delta$ is finite subloop of $\mathbb{O}^{*}$ since $(\omega x)(\omega y)=-y x^{*}=-y x^{-1}, x(\omega y)=$ $\omega\left(x^{*} y\right)=\omega\left(x^{-1} y\right)$ and $(\omega x) y=\omega(y x)$. Note that as a root system, $\Gamma=\Delta \oplus \omega \Delta$.

We call this procedure doubling of the loop $\Delta$ and $\Gamma$ the double of $\Delta$. Instead of $\omega$, we can use any element $\psi$ such that $n(\psi)=1$ and $\psi \perp \Gamma$, so the more precise term would be the double of $\Delta$ with respect to $\omega$.

Using the doubling procedure, we establish existence of subloops of types $A_{1}^{8}=$ $I_{2}(2)^{4}, I_{2}(n)^{4}(n>2), D_{4}^{2}, F_{4}^{2}, H_{4}^{2}$.

2.2. Subloop of type $E_{8}$. Let $\Gamma=\left\{ \pm e_{i},\left( \pm e_{i} \pm e_{j} \pm e_{k} \pm e_{l}\right) / 2\right\}$ for all possible $i$ and the following 14 subsets $\{i, j, k, l\}$ of $\{0,1,2,3,4,5,6,7\}$ :

$\begin{array}{llll}\{0,3,5,6\} & \{0,2,3,4\} & \{1,2,4,7\} & \{1,5,6,7\} \\ \{0,1,2,5\} & \{0,2,6,7\} & \{3,4,6,7\} & \{1,3,4,5\} \\ \{0,1,3,7\} & \{0,4,5,7\} & \{2,4,5,6\} & \{1,2,3,6\} \\ \{0,1,4,6\} & & \{2,3,5,7\} & \end{array}$

A tedious but straightforward calculation shows that it is a subloop with 240 elements. In fact, this loop is known as the loop of units in octavian integers [7].

This subloop must be a root system of either type $H_{4}^{2}$ or type $E_{8}$ (the only root systems of rank 8 with 240 elements). The type can be determined by $\Gamma^{(4)}$, characterised in terms of the root system. There are 126 elements of order 4 in $\Gamma$, and this is consistent with type $E_{8}$ ( $H_{4}^{2}$ would have 150 elements of order 4$)$. Alternatively, one can observe that $\Gamma^{(5)}$ is empty. This is true for $E_{8}$ but not $H_{4}^{2}$.

\section{Non-EXIstence}

In this section we show that the remaining root systems from the list do not appear among the subloops of $\mathbb{O}$. We need two following facts, the first of which is a version of Lagrange's theorem.

Lemma 6. If $x \in \Gamma$ and $\Gamma$ is a finite subloop of $\mathbb{O}$, then $|x|$ divides $|\Gamma|$.

Proof. Let $\Delta$ be the cyclic subgroup of $\Gamma$ generated by $x$. It suffices to show that cosets $\Delta z, z \in \Gamma$, are disjoint. Assume $\Delta z \cap \Delta w \neq \emptyset$. Then $x^{k} z \in \Delta z \cap \Delta w$ for some $k$. Thus, $\Delta z$ and $\Delta w$ are cosets of $\Delta$ in the group generated by $x$ and $z$, and $\Delta z=\Delta w$ by the standard argument.

Lemma 7. The root system $D_{4}$ cannot be embedded into the root system $A_{n}$ for any $n$. 
Proof. A root system $\Phi$ of type $A_{n}$ is constructed as follows. Start with an $n+1$ dimensional Euclidean space with orthonormal basis $\varepsilon_{0}, \cdots, \varepsilon_{n}$. Then take $\Phi$ to be the set of all vectors of the form $\frac{1}{\sqrt{2}}\left(\varepsilon_{i}-\varepsilon_{j}\right)$ for $i \neq j$. Suppose that a root system of type $D_{4}$ can be embedded in $\Phi$. Then, by considering only the simple roots of $D_{4}$, $\Phi$ must have a four-element subset $\left\{x, y_{1}, y_{2}, y_{3}\right\}$ with the $y_{i}$ pairwise orthogonal and with $\left\langle x, y_{i}\right\rangle=-\frac{1}{2}$. Without loss of generality we may assume $x=\frac{1}{\sqrt{2}}\left(\varepsilon_{0}-\varepsilon_{1}\right)$. Then we have

$$
\left\{y \in \Phi \mid\langle x, y\rangle=-\frac{1}{2}\right\}=\left\{\frac{1}{\sqrt{2}}\left(\varepsilon_{i}-\varepsilon_{0}\right) \mid i>1\right\} \cup\left\{\frac{1}{\sqrt{2}}\left(\varepsilon_{1}-\varepsilon_{i}\right) \mid i>1\right\} .
$$

But no two vectors in either one of the two sets on the right are orthogonal. Therefore we have contradicted the pigeonhole principle.

3.1. Subloops of type $A_{4}^{2}, B_{4}^{2}$. Let $\Gamma$ be a subloop of one of these two types. Let us write $\Gamma=\Gamma_{1} \oplus \Gamma_{2}$ (as a root system), where $\Gamma_{i}$ is an irreducible root system of rank 4 and $1 \in \Gamma_{1}$. By Lemma $2, \Gamma_{1}$ is a subloop so it has to be a subgroup of a copy of $\mathbb{H}$ - but subgroups of type $A_{4}$ and $B_{4}$ do not exist.

3.2. Subloop of type $B_{8}$. Note that in $B_{8}$ some elements have 98 orthogonal elements (root system $B_{7}$ ) while others have just 74 (root system $B_{6} \oplus A_{1}$ ). This is impossible since multiplication by an element $g \in \Gamma$ is an orthogonal bijection of $\Gamma$.

3.3. Subloop of type $A_{8}$. The order of such a subloop $\Gamma$ would be $72=2^{3} 3^{2}$. Roots in $A_{8}$ orthogonal to $e$ form $A_{6}$. So, $\left|\Gamma^{(4)}\right|=42$. The possible values of $\cos (\alpha)$ for an angle between roots in $A_{8}$ are $0, \pm 1 / 2, \pm 1$. This implies that possible orders of elements in $\Gamma$ are 1, 2, 3, 4 and 6. In fact, $\left|\Gamma^{(3)}\right|=\left|\Gamma^{(6)}\right|=14$.

Let us pick $x, y \in \Gamma^{(3)}$ such that $x \neq y$ and $x \neq y^{-1}$. Let $\Delta$ be the subloop generated by $x$ and $y$. Since $\Delta$ is non-commutative and associative, it is a root system of one of the types $I_{2}(k)^{2}, k \geq 2, D_{4}, F_{4}, H_{4}$. Since possible orders of elements in $\Delta$ are $1,2,3,4,6$, it is a root system of one of the types $I_{2}(2)^{2}, I_{2}(3)^{2}$, $D_{4}$.

The contradiction to the existence of $\Gamma$ is that each of these cases is impossible. The group $I_{2}(2)^{2}$ is of order 8 and has no elements of order 3 at all. The group $I_{2}(3)^{2}$ is the binary dihedral of order 12 . It has just 2 elements of order 3 , and they are inverses of each other. Finally the group $D_{4}$ is impossible since the root system $D_{4}$ cannot be embedded into $A_{n}$ (Lemma 7 ).

3.4. Subloop of type $D_{8}$. The order of such a subloop $\Gamma$ would be $112=2^{4} 7$. By inspecting the root system, $\left|\Gamma^{(3)}\right|=24$. However, existence of elements of order 3 contradicts Lemma 6 .

\section{Conjugacy}

Now we are ready to finish the proof of the main theorem by showing that two non-associative subloops of the same type as root systems are conjugate by an automorphism.

Lemma 8. Let $\Gamma$ and $\Delta$ be non-associative finite subloops of $\mathbb{O}$. If $\Gamma$ and $\Delta$ are root systems of the same type, then there exists $g \in G$ such that $g(\Gamma)=\Delta$. 
Proof. We know what the possible types of root systems $\Gamma$ and $\Delta$ are. Let us consider the two cases.

Case 1. Let us assume that both $\Gamma$ and $\Delta$ are of type $X^{2}$ where $X$ is a root system of rank 4 . We first prove that $\Gamma$ can be written as $\Gamma_{1} \oplus \Gamma_{2}$, where the $\Gamma_{i}$ are orthogonal root systems of type $X$ and $\Gamma_{1}$ is a subloop. Let $t \in\{2,4,8\}$ be the number of irreducible components of $\Gamma$. If $t=2$ the result is immediate from Lemma 2. If $t=8$, then $\Gamma \cong A_{1}^{8}$, so the irreducible component containing 1 is $\{1,-1\}$. Pick any 2 elements $x, y$ in $\Gamma$ which do not commute. Then $x, y$ generate (as an algebra) a copy of $\mathbb{H}$ and hence a loop which is of the required form by the final statement of Lemma 2. If $t=4$, then the irreducible component containing 1 is a cyclic group $\langle x\rangle$. Pick any $y \in \Gamma$ not commuting with $x$. Then, as before, the subloop generated by $x, y$ has the required form.

Repeat the same argument for $\Delta$. Since $G$ acts transitively on copies of $\mathbb{H}$ in $\mathbb{O}$ (Lemma 3) there exists $g_{1} \in G$ such that $g_{1} \mathbb{R} \Gamma_{1}=\mathbb{R} \Delta_{1}$. Since an automorphism of $\mathbb{H}$ can be extended to an automorphism of $\mathbb{O}$, by the classification of subloops of $\mathbb{H}$, we can find $g_{2} \in G$ such that $g_{2} g_{1} \Gamma_{1}=\Delta_{1}$. Now pick $x \in g_{2} g_{1} \Gamma_{2}$ and $y \in \Delta_{2}$. Since the stabilizer of $\mathbb{H}$ in $G$ acts transitively on the unit sphere in the orthogonal complement of $\mathbb{H}$ we can find $g_{3} \in \operatorname{Stab}_{G}\left(\Delta_{1}\right)$ such that $g_{3} x=y$. Clearly, $g_{3} g_{2} g_{1} \Gamma=\Delta$.

Case 2. Let us assume that both $\Gamma$ and $\Delta$ are of type $E_{8}$. There exists a subloop of type $A_{1}^{8}$ in both of them. Indeed, elements orthogonal to 1 in $\Gamma$ form the root system $E_{7}$. Pick one such element, call it $I$. It generates a cyclic subgroup $\Gamma_{1}$ of order 4 that is a root system $A_{1}^{2}$. Elements orthogonal to $I$ in this $E_{7}$ form $D_{6}$. Pick such an element $J$. Doubling $\Gamma_{1}$ with respect to $J$ gives a subloop $\Gamma_{2}=\Gamma_{1} \oplus J \Gamma_{1}$ that is a root system $A_{1}^{4}$. Elements orthogonal to $J$ in $D_{6}$ form the root system $A_{1} \oplus D_{4}$. Pick an element $\omega$ there that lies in a different component than $J I$. Since $\omega$ orthogonal to $\Gamma_{2}$, one can again double to arrive at a subloop $\Gamma_{2} \oplus \omega \Gamma_{2}$ that is a root system $A_{1}^{8}$.

By Case 1 , there exists $g_{1} \in G$ that sends the $A_{1}^{8}$ subloop of $\Gamma$ to the $A_{1}^{8}$ subloop $\Delta_{3}$ of $\Delta$. Let us consider the $\mathbb{Z}$-spans $\mathbb{Z}\left(g_{1} \Gamma\right)$ and $\mathbb{Z} \Delta$. These are the rings of integers containing the same ring of Gravesian integers [7]. To establish this one needs to prove that $\mathbb{Z} \Delta \subseteq \mathbb{Q} \Delta_{3}, 2 \operatorname{Re}(\mathbb{Z} \Delta) \subseteq \mathbb{Z}$ and $\langle x, x\rangle \in \mathbb{Z}$ for all $x \in \mathbb{Z} \Delta$. All of this easily follows from the fact that $E_{8}$ is simply-laced.

Both $\mathbb{Z}\left(g_{1} \Gamma\right)$ and $\mathbb{Z} \Delta$ are octavian integers since the loops of units contain $E_{8}$. Thus, there exists $g_{2} \in G$ such that $g_{2} \mathbb{Z}\left(g_{1} \Gamma\right)=\mathbb{Z} \Delta[7$. Since $\Delta$ is the loop of units in $\mathbb{Z} \Delta$, we conclude that $g_{2} g_{1} \Gamma=\Delta$ [7, 10.4].

\section{Question}

We believe that these finite loops could give rise to new interesting geometric objects.

Let $\Gamma$ be a finite subloop of $\mathbb{O}$. Let $R(\Gamma)$ be the finite group generated by right multiplications by elements of $\Gamma$. It acts on $\mathbb{O}$. Let $X$ be a minimal (in a suitable sense) resolution of the quotient $\mathbb{O} / R(\Gamma)$. Does $X$ admit a Riemannian structure with $\operatorname{Spin}_{7}$-holonomy?

\section{REFERENCES}

[1] C. Jordan, Memoire sur les equations differentielles lineaires a integrale algebrique, J. Reine Angew. Math., 84 (1878), 89-215. 
[2] J. McKay, Graphs, singularities, and finite groups, in Santa Cruz conference on finite groups, Proc. Symp. Pure Math. 37, 1980, pp 183-186. MR0604577 (82e:20014)

[3] R. T. Curtis, A classification and investigation of the finite subloops of the Cayley-Dickson algebra, manuscript, 1970.

[4] R. T. Curtis, Construction of a family of Moufang loops, Math. Proc. Cambridge Philos. Soc., to appear.

[5] Y. Ito, I. Nakamura, Hilbert schemes and simple singularities. New trends in algebraic geometry (Warwick, 1996), 151-233, Cambridge University Press, Cambridge, 1999. MR1714824 (2000i:14004)

[6] J. Humphreys, Reflection Groups and Coxeter Groups. Cambridge University Press, Cambridge, 1990. MR $1066460(92 \mathrm{~h}: 20002)$

[7] J. Conway, D. Smith, On quaternions and octonions: their geometry, arithmetic, and symmetry. A K Peters, Natick, 2003. MR1957212 (2004a:17002)

Department of Mathematics, University of Warwick, Coventry, CV4 7AL, United KINGDOM

E-mail address: paulsboddington@yahoo.co.uk

Department of Mathematics, University of Warwick, Coventry, CV4 7AL, United KINGDOM

E-mail address: rumynin@maths.warwick.ac.uk 